\title{
NONRATIONAL HYPERSURFACES
}

\author{
JÁNOS KOLLÁR
}

Let $X_{d} \subset \mathbb{P}^{n+1}$ be a smooth hypersurface. If $d \geq n+2$ then $X_{d}$ contains very few rational curves. If $d \leq n+1$ then $X_{d}$ is covered by rational curves, in fact any two points can be connected by a rational curve in $X_{d}$. The best known algebraic variety with lots of rational curves is $\mathbb{P}^{n}$, and early on the question was raised if these hypersurfaces are birational to $\mathbb{P}^{n}$ or not.

It is easy to see that $X_{d}$ is rational if $d=2$ (project $X_{d}$ from any of its points). It is less clear, but has been know for a hundred years, that any smooth cubic in $\mathbb{P}^{3}$ is rational.

1. Definition. Let $X$ be a variety of dimension $n$. We say that $X$ is rational (resp. unirational) if there is a map $\mathbb{P}^{n} \rightarrow X$ which is birational (resp. dominant).

We say that $X$ is ruled (resp. uniruled) if there is a variety $Y$ of dimension $n-1$ and a map $Y \times \mathbb{P}^{1} \rightarrow X$ which is birational (resp. dominant).

In positive characteristics we say that $X$ is separably unirational (resp. separably uniruled) if the above map is also separable.

The rationality question for threefolds of degrees 3 and 4 in $\mathbb{P}^{4}$ has been open for a long time. In the early seventies two different methods were discovered. Together they settled the question completely.

Iskovskikh and Manin [5] developed the Nöther-Fano method which enabled them to prove that any birational self-map of any smooth quartic is an isomorphism. This in particular implies that they are not rational. The same method shows that they are not birational to conic bundles or to a family of Del Pezzo surfaces. This approach was further developped and applied to many other threefolds by Iskovskikh and his students (see, e.g., [4, 10, 11]). This method in principle works in any dimension, but the technical difficulties seem rather serious and have been overcome in a few cases only. Pukhlikov [9] proves that any birational self-map of a smooth quintic in $\mathbb{P}^{5}$ is an isomorphism.

Clemens and Griffiths [3] introduced the method of studying the intermediate Jacobian and used it to show that smooth cubic threefolds are not rational. This method has been very successful in dimension three (see, e.g., [2]). Unfortunately it does not work in higher dimensions, at least not in the usual formulation.

Received by the editors March 2, 1994.

1991 Mathematics Subject Classification. Primary 14J45, 14J70, 14M20.

Key words and phrases. Rational varieties, ruled varieties, Fano varieties. 
Artin and Mumford [1] observed that the torsion subgroup of $H^{3}(X, \mathbb{Z})$ is a birational invariant and used this to find examples of nonrational but unirational varieties in any dimension.

The aim of this article is to develop a rather simple method which provides many examples of Fano varieties which are not rational. The method in fact shows that they are not even ruled, which is a considerably stronger result in higher dimensions. Applied to hypersurfaces, it gives the following:

2. Theorem. Let $X_{d} \subset \mathbb{P}^{n+1}$ be a very general hypersurface over $\mathbb{C}$ of degree

$$
d \geq \frac{2}{3}(n+3) \text {. }
$$

Assume in addition that $n$ and $d$ are both even. Then $X_{d}$ is not ruled.

2.1. Remark. The assumptions that $n$ and $d$ are both even are only for convenience of proof. The result holds without them (see (4.1)).

3. Comments. (3.1) Here "very general" means that the result holds for hypersurfaces corresponding to a point in the complement of countably many closed subvarieties in the space of all hypersurfaces. The proof does not give any method of determining these closed subvarieties. However, it is possible to write down examples of hypersurfaces defined over $\mathbb{Q}$ which are shown to be nonruled by a slight variation of the method.

It is quite likely that any smooth hypersurface whose degree satisfies the above condition is nonruled.

(3.2) The result is of interest only if $d \leq n+1$, since a smooth hypersurface of degree at least $n+2$ is not even uniruled.

(3.3) It is not clear what one should expect about the rationality question of hypersurfaces. Any quadric is rational. If a cubic $X_{3} \in \mathbb{P}^{2 n+1}$ contains two skew linear spaces of dimension $n$ then it is rational. (There are such smooth cubics.) It is not known what happens with the general cubic in $\mathbb{P}^{5}$. I do not know any results for higher degrees.

The method can be refined in several ways to give more precise information. Here I would like to concentrate on the main idea and leave the proofs of the following assertions for a later occasion. ( $\left.{ }^{r} x\right\urcorner$ denotes the smallest integer $\geq x$.)

4. Theorem. Let $X_{d} \subset \mathbb{P}^{n+1}$ be a very general hypersurface over $\mathbb{C}$.

(4.1) If

$$
d \geq 2 r(n+3) / 3^{r}
$$

then $X_{d}$ is not ruled.

(4.2) If

$$
d \geq 3\ulcorner(n+3) / 4\urcorner
$$

then $X_{d}$ is not birational to a conic bundle.

(4.3) Assume that $d=n+1$. Let $Y$ be a variety of dimension $n-1$ and $\phi: Y \times \mathbb{P}^{1} \rightarrow X_{d}$ a dominant map. Then $\operatorname{deg} \phi$ is divisible by every prime less than $\sqrt{n}$. 
The method of proof is by degeneration. Instead of finding directly a smooth hypersurface which is not ruled, I find a variety which is not ruled and which can be obtained as a limit of a family of hypersurfaces. The examples are ramified covers of hypersurfaces over a field of positive characteristic. The required degeneration is given by the following:

5. Construction (Mori $[8,4.3]$ ). Let $S$ be a scheme, $t \in \mathscr{O}_{S}$. Let $f, g \in$ $\mathscr{O}_{S}\left[x_{0}, \ldots, x_{n}\right]$ be homogeneous polynomials of degrees $c d$ and $d$ respectively such that $g^{c}-f$ is not identically zero $\bmod s$ for any point $s \in S$. The scheme

$$
Z=\left(y^{c}-f=t y-g=0\right) \subset \mathbb{P}_{S}\left(x_{0}, \ldots, x_{n}, y\right)
$$

defines a family of weighted complete intersections over $S$. If $s \in S$ and $t(s) \neq 0$ then the fiber of $Z \rightarrow S$ over $s$ is isomorphic to the hypersurface

$$
\left(g(s)^{c}-t(s)^{c} f(s)=0\right) \subset \mathbb{P}_{s}\left(x_{0}, \ldots, x_{n}\right) .
$$

If $t(s)=0$ then the fiber of $Z \rightarrow S$ over $s$ is isomorphic to a degree $c$ cover of the hypersurface $(g(s)=0)$ ramified along $(f(s)=0)$ (cf. Construction 8).

The degeneration method works thanks to the following result of Matsusaka [7, Appendix 1.1]. See [6, IV.1.8.4] for a detailed proof and for further results.

6. Theorem [7]. Let $f: X \rightarrow S$ be a flat proper morphism with irreducible and reduced geometric fibers. Then there are countably many closed subvarieties $S_{i} \subset S$ such that for any closed point $s \in S$ the fiber $f^{-1}(s)$ is ruled over the algebraic closure of the residue field $k(s)$ iff $s \in \cup S_{i}$.

Going to positive characteristics seems an essential feature of the proof. It is expected that the tangent bundle of any hypersurface is stable (even more, they are expected to have a Kähler-Einstein metric). In positive characteristics stability can fail in a rather strong way. Sufficiently strong instability implies that the variety is not ruled and not even separably uniruled:

7. Lemma. Let $X$ be a smooth proper variety and $M$ a line bundle on $X$ such that global sections of $M^{m}$ define a birational map for some $m>0$. Assume that there is an injection $M \rightarrow \wedge^{i} \Omega_{X}^{1}$ for some $i>0$.

Then $X$ is not ruled. Moreover, if $Y$ is any variety of dimension $n-1$ and $\phi: Y \times \mathbb{P}^{1} \rightarrow X$ a dominant map then $\phi$ is not separable.

Proof. By shrinking $Y$ we may assume that $\phi$ is a morphism. We have a natural map

$$
T_{Y \times \mathbb{P}^{1}} \rightarrow \phi^{*} T_{X},
$$

which is generically an isomorphism if $\phi$ is separable. Let $\phi_{y}: \mathbb{P}^{1} \rightarrow X$ denote the restriction of $\phi$ to $\{y\} \times \mathbb{P}^{1}$. For general $y$ we obtain an injection

$$
\mathscr{O}_{\mathbb{P}^{1}}^{n-1} \times \mathscr{O}_{\mathbb{P}^{1}}(2) \hookrightarrow \phi_{y}^{*} T_{X},
$$

which is generically an isomorphism. Taking the dual and wedge product we obtain an injection

$$
\phi_{y}^{*} M \hookrightarrow \wedge^{i} \phi_{y}^{*} \Omega_{X}^{1} \hookrightarrow \wedge^{i}\left(\mathscr{O}_{\mathbb{P}^{1}}^{n-1} \times \mathscr{O}_{\mathbb{P}^{1}}(-2)\right) .
$$


By assumption $\phi_{y}^{*} M$ has positive degree, which is a contradiction.

7.1. Remark. By the Bogomolov-Sommese vanishing theorem the assumptions of Lemma 7 can be satisfied in characteristic zero only if $i=\operatorname{dim} X$. In this case $X$ is of general type and not even uniruled.

It is time to get down to the construction of the examples.

8. Construction. Let $X$ be a scheme and $L$ a line bundle on $X$. Let $\pi: U \rightarrow$ $Z$ denote the total space of $L$, i.e., $\pi_{*} \mathscr{O}_{U}=\sum_{m \geq 0} L^{-m}$. Let $s \in H^{0}\left(X, L^{k}\right)$ for some fixed $k$.

$\pi_{*}\left(\pi^{*} L\right)=\sum_{m \geq-1} L^{-m}$, hence $\pi^{*} L$ has a canonical section corresponding to $1 \in H^{0}\left(X, L^{0} \cong \mathscr{O}_{X}\right)$. Denote this section by $y_{L}$. Since $\pi_{*}\left(\pi^{*} L^{k}\right)=$ $\sum_{m \geq-k} L^{-m}$, both $y_{L}^{k}$ and $s$ can be viewed as sections of $\pi^{*} L^{k}$. Let $Y:=$ $\left(y_{L}^{k}-s\right) \subset U$ be the zero set. The restriction of $\pi$ to $Y$ is also denoted by $\pi$. $Y$ is called the covering of $X$ obtained by taking the $k^{\text {th }}$ root of $s$. It is also denoted by $X[\sqrt[k]{s}]$.

Choose local coordinates $x_{i}$ at a point $x \in X$. On $U$ we can use $y:=y_{L}$ and the $x_{i}$ as local coordinates. Locally $Y$ is given by the equation $y^{k}-$ $s\left(x_{1}, \ldots, x_{n}\right)=0$.

The following properties are immediate from the definition:

9. Lemma. Notation as above.

(9.1) There is an exact sequence $0 \rightarrow \pi^{*} \Omega_{X}^{1} \rightarrow \Omega_{U}^{1} \rightarrow \pi^{*} L^{-1} \rightarrow 0$.

(9.2) $\mathscr{O}_{U}(-Y) \cong \pi^{*} L^{-k}$.

(9.3) There is an exact sequence $\pi^{*} L^{-k} \stackrel{d_{Y}}{\longrightarrow} \Omega_{U}^{1} \rightarrow \Omega_{Y}^{1} \rightarrow 0$. In the above local coordinates the image of $d_{Y}$ is given by

$$
\left(-\frac{\partial s}{\partial x_{1}} d x_{1}, \ldots,-\frac{\partial s}{\partial x_{n}} d x_{n}, k y^{k-1} d y\right) .
$$

The map $d_{Y}$ in (9.3) becomes very special if the characteristic $p$ of the base field divides $k$. In this case $k y^{k-1} d y=0$ and so $\operatorname{im} d_{Y} \subset \pi^{*} \Omega_{X}^{1}$. Thus we obtain the exact sequence

$$
0 \rightarrow \operatorname{coker}\left[\pi^{*} L^{-k} \stackrel{d_{Y}}{\longrightarrow} \pi^{*} \Omega_{X}^{1}\right] \rightarrow \Omega_{Y}^{1} \rightarrow \pi^{*} L^{-1} \rightarrow 0 .
$$

10. Idea of the proof of Theorem 2. Choose $X$ to be a Fano variety and $L$ ample. Construct $Y$ as above. Then $K_{Y}=\pi^{*}\left(K_{X}+(k-1) L\right)$; thus $Y$ is Fano if $K_{X}+(k-1) L$ is negative. In (9.4) $\Omega_{Y}^{1}$ is expressed as an extension and the corresponding first Chern classes are

$$
\pi^{*}\left(K_{X}+k L\right) \text { and } \pi^{*}(-L) .
$$

In some cases one can choose $X$ and $L$ such that $K_{X}+(k-1) L$ is negative (so $Y$ is Fano), but $K_{X}+k L$ is positive; thus $\Omega_{Y}^{1}$ is very unstable.

Unfortunately the constructed cyclic cover $Y$ is almost never smooth. We need to understand the singularities of $Y$ and to see how the sequence (9.4) can be lifted to a smooth model of $Y$. 
The precise technical result is the following:

11. Theorem. Let $X$ be a smooth projective variety over a field of char $p$ and $L$ a line bundle on $X$. Assume that:

(11.1) For every closed point $x \in X$ the restriction map

$$
H^{0}\left(X, L^{p}\right) \rightarrow\left(\mathscr{O}_{X} / m_{x}^{3}\right) \otimes L^{p}
$$

is surjective, where $m_{x}$ is the ideal sheaf of $x \in X$. (In characteristic 2 assume in addition that $\operatorname{dim} X$ is even.)

(11.2) $L^{p} \otimes K_{X}$ is ample.

Then for general $s \in H^{0}\left(X, L^{p}\right)$ the corresponding p-fold cover $Y=X[\sqrt[p]{s}]$ is not separably uniruled.

11.3. Remarks. The condition (11.1) and the general choice of $s$ are used to guarantee that $Y$ has "simple" singularities. There are many other singularities where the lifting in $\S 22$ is still possible. This is useful if one wants to get nice concrete examples of nonruled hypersurfaces.

The assumption about even dimensionality in char 2 is again not necessary. A more careful analysis of the generic critical points can be done easily.

12. Proof of Theorem 2. Pick a prime $p$. By Construction 5 there is a family of degree $p d$ hypersurfaces degenerating to a general $p$-fold cover of a degree $d$ hypersurface in characteristic $p$ ramified along a divisor of degree $p d$. Condition (11.1) is always satisfied, and (11.2) becomes $p d+d-n-2>0$. Taking $p=2$ gives Theorem 2 .

The proof of Theorem 11 is done in several steps.

In order to understand the first term in (9.4) better, we need a simple observation.

13. Definition-Lemma. Let $X$ be a smooth variety over a field of char $p$ and $L$ a line bundle on $X$. If $p \mid k$ then there is a natural differential

$$
d: L^{k} \rightarrow L^{k} \otimes \Omega_{X}^{1},
$$

constructed as follows. Let $\tau$ be a local generator of $L, s=f \tau^{k}$ a local section of $L^{k}$, and the $x_{i}$ local coordinates. Set

$$
d(s):=\sum \frac{\partial f}{\partial x_{i}} \tau^{k} d x_{i} .
$$

This is independent of the choices made and thus defines $d$. once:

Comparing the definitions of $d_{Y}$ in (9.4) and of $d$ in (13.1) we obtain at

14. Lemma. Notation as above.

(14.1) For a fixed $s \in H^{0}\left(X, L^{k}\right)$ we can view $d(s)$ as a sheaf homomorphism $d(s): \mathscr{O}_{X} \rightarrow L^{k} \otimes \Omega_{X}^{1}$. Tensoring with $L^{-k}$ we obtain

$$
d s: L^{-k} \rightarrow \Omega_{X}^{1} \text {. }
$$


(14.2) $d_{Y}=-\pi^{*} d s$; thus there is an exact sequence

$$
0 \rightarrow \pi^{*} \text { coker }\left[L^{-k} \stackrel{d s}{\longrightarrow} \Omega_{X}^{1}\right] \rightarrow \Omega_{Y}^{1} \rightarrow \pi^{*} L^{-1} \rightarrow 0 .
$$

Instead of trying to deal with the coherent sheaf coker $\left[L^{-k} \stackrel{d s}{\longrightarrow} \Omega_{X}^{1}\right]$, it is easier to deal with a line bundle:

15. Definition. Notation as above. Assume in addition that $d s$ is nonzero in codimension one. Let $Q(L, s)$ denote the double dual of the determinant of $\operatorname{coker}\left[L^{-k} \stackrel{d s}{\longrightarrow} \Omega_{X}^{1}\right]$.

$Q(L, s)$ is a line bundle on $X$ and $c_{1}(Q(L, s))=K_{X}+k L$. By construction there are natural maps

$$
q: \wedge^{n-1} \Omega_{X}^{1} \rightarrow Q(L, s)
$$

and

$$
\pi^{*} Q(L, s) \hookrightarrow\left(\wedge^{n-1} \Omega_{Y}^{1}\right)^{* *} .
$$

Let $r: Y^{\prime} \rightarrow Y$ be a resolution of singularities. We would like to prove that (15.2) lifts to an injection

$$
r^{*} \pi^{*} Q(L, s) \rightarrow \wedge^{n-1} \Omega_{Y^{\prime}}^{1}
$$

This question is local above the singular points of $Y$ and will be settled by an explicit computation once the singular points of $Y$ and the behaviour of $Q(L, s)$ near them are sufficiently well understood.

16. Lemma. Notation as above. Write $s=f \tau^{k}$ and let

$$
\eta_{i}:=\frac{d x_{1} \wedge \cdots \wedge \widehat{d x_{i}} \wedge \cdots \wedge d x_{n}}{\partial f / \partial x_{i}}
$$

( $\eta_{i}$ is undefined if $\partial f / \partial x_{i}$ is identically zero).

Then $q\left(\eta_{i}\right)= \pm q\left(\eta_{j}\right)$ and they give local generators of $Q(L, s)$.

Proof. By definition the image of $d s$ is generated by $\sum_{i}\left(\partial f / \partial x_{i}\right) d x_{i}$. This implies that $q\left(\eta_{i}\right)= \pm q\left(\eta_{j}\right)$ and that $\eta_{i}$ is a local generator of $Q(L, s)$ at $x \in X$ if $\partial f / \partial x_{i}$ is nonzero at $x$. Thus $q\left(\eta_{i}\right)$ is a local generator outside a codimension two set, hence everywhere.

In order to understand the singularities of $Y$, we need the following:

17. Definition-Lemma. Let $X$ be a smooth variety over a field of char $p$ and $L$ a line bundle on $X$. Let $k$ be an integer divisible by $p$.

(17.1) We say that a local section $s$ of $L^{k}$ has a critical point at $x \in X$ if $d(s) \in \Gamma\left(L^{k} \otimes \Omega_{X}^{1}\right)$ vanishes at $x$.

(17.2) Pick local coordinates $x_{i}$ near $x$ and a local generator $\tau$ of $L$ at $x$. Write $s=f \tau^{k}$. The matrix

$$
H(s):=\left(\frac{\partial^{2} f}{\partial x_{i} \partial x_{j}}\right)
$$


is called the Hessian of $s$ with respect to the coordinates $x_{i}$ and the generator $\tau$. The rank of $H(s)$ at a point $x \in X$ is independent of the choices of $x_{i}$ and $\tau$.

(17.3) The critical point of $s$ at $x$ is called nondegenerate if rank $H(s)(x)=$ $\operatorname{dim} X$.

17.4. Remark. If char $=2$ and $\operatorname{dim} X$ is odd then every critical point is degenerate since every quadratic form in an odd number of variables is degenerate in char 2 . In the other cases it is $\operatorname{dim} X+1$ condition for a section to have a degenerate critical point at a given $x \in X$.

By a simple dimension count we obtain:

18. Proposition (algebraic Morse lemma). Let $X$ be a smooth variety over a field of char $p$ and $L$ a line bundle on $X$. Let $k$ be an integer divisible by $p$ and $W \subset H^{0}\left(X, L^{k}\right)$ a finite dimensional subvectorspace. Assume that for every closed point $x \in X$ the restriction map

$$
W \rightarrow\left(\mathscr{O}_{X} / m_{x}^{3}\right) \otimes L^{k}
$$

is surjective, where $m_{x}$ is the ideal sheaf of $x \in X$. In characteristic 2 assume in addition that $\operatorname{dim} X$ is even.

Then a general section $f \in W$ has only nondegenerate critical points.

19. Definition. For simplicity of notation we denote the higher order terms in an expression by h.o.t. Thus the expression $f(x, y)=f_{2}+$ h.o. $\mathrm{t}_{x}(x, y)$ means that $f_{2}$ is a degree two homogeneous polynomial and in h.o.t $\mathrm{t}_{x}(x, y)$ the degree of every monomial is at least 3 in $x$ (e.g., $x^{2} y^{5}$ is not allowed in h. o. $\mathrm{t}_{x}(x, y)$ of this example).

20. The singularities of $Y=X[\sqrt[p]{s}]$. Using the above notation assume for simplicity that $k=p$. The local equation of $Y$ has the form

$$
y^{p}-f\left(x_{1}, \ldots, x_{n}\right)=0 \text {. }
$$

We write $f=f_{0}+f_{1}+f_{2}+$ h. o. $\mathrm{t}(x)$ where $f_{i}$ is homogeneous of degree $i$. Replacing $y$ by $y-\sqrt[p]{f_{0}}$ the equation becomes

$$
y^{p}=f_{1}+f_{2}+\text { h. o. } \mathrm{t}(x) .
$$

If $f_{1} \neq 0$ then $Y$ is smooth at the origin. Thus the singular points of $Y$ correspond to the critical points of $s$ and they have local equations

$$
y^{p}=f_{2}+\text { h. o. } \mathrm{t}(x) \text {. }
$$

21. Resolution of the singularities of $Y$. Let $W$ be an $n+1$ dimensional variety with a local coordinate system $y, x_{1}, \ldots, x_{n}$. I think of $y$ as being distinguished, the $x$ coordinates are changeable. Consider a hypersurface singularity given by a local equation

$$
Y=\left(y^{k}=f_{2}(x)+\text { h. o. } \mathrm{t}_{x}(x, y)\right)
$$

where $f_{2}$ is a nondegenerate quadric in the $x$ variables. $Y$ has an isolated singularity at the origin. Blow up the origin to obtain $b: B_{0} W \rightarrow W$ and let 
$b: B_{0} Y \rightarrow Y$ denote the blow up of $Y . B_{0} Y$ still has at most one singular point corresponding to the line $x_{1}=\cdots=x_{n}=0$. Use the substitutions

$$
y:=y \quad \text { and } \quad x_{i}:=x_{i} / y
$$

to obtain new local coordinates on $B_{0} W$. The equation of $B_{0} Y$ in this coordinate system is

$$
y^{k-2}=f_{2}(x)+\text { h. o. } \mathrm{t}_{x}(x, y) \text {. }
$$

The exceptional divisor of $b: B_{0} Y \rightarrow Y$ is locally given by $y=0$. After finitely many such steps the singularity of $Y$ is resolved.

22. Lifting of $\eta_{i}$. Choosing the $x$-coordinates on $W$ suitably we may assume that

$$
f_{2}=x_{n} x_{n-1}+\bar{f}_{2}\left(x_{1}, \ldots, x_{n-2}\right) .
$$

With this choice of coordinates let

$$
\eta_{n}=\frac{d x_{1} \wedge \cdots \wedge d x_{n-1}}{x_{n-1}+\operatorname{h.o.t}(x)}
$$

For inductive purposes it is convenient to introduce the following:

22.3. Definition. We say that a (meromorphic) differential form on $W$ is of type eta (with respect to a choice of a local coordinate system) if it can be written as a linear combination (with regular coefficients) of the differential forms

$$
\frac{d x_{1} \wedge \cdots \wedge d x_{n-1}}{x_{n-1}+\text { h. o. } \mathrm{t}_{x}(x, y)} \text { and } \frac{d x_{1} \wedge \cdots \wedge \widehat{d x}_{i} \wedge \cdots \wedge d x_{n-1} \wedge d y}{x_{n-1}+\text { h. o. } \mathrm{t}_{x}(x, y)}
$$

22.4. Lemma. Notation as above. Assume that $n \geq 3$. Let $\omega$ be an $(n-1)$ form of type eta on $W$. Then $b^{*} \omega$ is also of type eta on $B_{0} W$ (with respect to the new coordinate system (21.2)).

Proof. Computing in the first case we obtain that

$$
\begin{aligned}
& b^{*} \frac{d x_{1} \wedge \cdots \wedge d x_{n-1}}{x_{n-1}+\text { h.o.t } x(x, y)}=\frac{d\left(y x_{1}\right) \wedge \cdots \wedge d\left(y x_{n-1}\right)}{y x_{n-1}+y^{2} \text { h.o.t } x(x, y)} \\
& =y^{n-2} \frac{d x_{1} \wedge \cdots \wedge d x_{n-1}}{x_{n-1}+\text { h. o. } \mathrm{t}_{x}(x, y)}+\sum_{i} \pm y^{n-3} \frac{d x_{1} \wedge \cdots \wedge \widehat{d x}_{i} \wedge \cdots \wedge d x_{n-1} \wedge d y}{x_{n-1}+\text { h. o. } \mathrm{t}_{x}(x, y)} \text {. }
\end{aligned}
$$

The computation in the other cases is analogous.

23. Proof of Theorem 11. Choose a general $s \in H^{0}\left(X, L^{p}\right)$. By Proposition $18 s$ has only nondegenerate critical points, hence the corresponding cover $Y=X[\sqrt[2]{s}]$ has only singular points as described in $\S 21$. These can be resolved by the process explained there. Let $r: Y^{\prime} \rightarrow Y$ be the resulting resolution of singularities.

As in Definition 15 we need to show that

$$
\pi^{*} Q(L, s) \rightarrow\left(\wedge^{n-1} \Omega_{Y}^{1}\right)^{* *} \quad \text { lifts to an injection } r^{*} \pi^{*} Q(L, s) \rightarrow \wedge^{n-1} \Omega_{Y^{\prime}}^{1}
$$


Choose local coordinates as in (22.1) and let $\eta_{n}$ be as in (22.2). By Lemma 16 $q\left(\eta_{n}\right)$ is a local generator of $Q(L, s) . q\left(\eta_{n}\right)$ lifts to a meromorphic differential form on $Y^{\prime}$ and we only need to show that it does not have a pole along any of the exceptional divisors of $r$.

This can be checked one blow up at a time. The exceptional divisor of one blow up is given by $y=0$, and by Lemma $22.4 b^{*} \eta_{n}$ is generically regular along $(y=0)$, hence so is $q\left(\eta_{n}\right)$. Thus we obtain an injection $r^{*} \pi^{*} Q(L, s) \rightarrow$ $\wedge^{n-1} \Omega_{Y^{\prime}}^{1}$. Set $M=r^{*} \pi^{*} Q(L, s)$.

$c_{1}(M)=r^{*} \pi^{*}\left(K_{X}+p L\right)$, hence by (11.2) a suitable power of $M$ determines a linear system whose image is $Y$. Thus Lemma 7 implies Theorem 11.

\section{ACKNOWLEDGEMENTS}

I would like to thank the referee for correcting some mistakes of the original version.

Partial financial support was provided by the National Science Foundation under grant numbers DMS-8707320 and DMS-9102866.

\section{REFERENCES}

1. M. Artin and D. Mumford, Some elementary examples of uniruled varieties which are not rational, Proc. London. Math. Soc. 25 (1972), 75-95.

2. A. Beauville, Variété de Prym et Jacobiennes intermédiaire, Ann. Sci. École Norm. Sup. 10 (1977), 309-391.

3. H. Clemens and P. Griffiths, The intermediate Jacobian of the cubic threefold, Ann. of Math. (2) 95 (1972), 281-356.

4. V. A. Iskovskikh, Birational automorphisms of three-dimensional algebraic varieties, J. Soviet Math. 13 (1980), 815-868.

5. V. A. Iskovskikh and Ju. I. Manin, Three-dimensional quartics and counterexamples to the Lüroth problem, Math. USSR-Sb. 15 (1971), 141-166.

6. J. Kollár, Rational curves on algebraic varieties (in preparation), 1994.

7. T. Matsusaka, Algebraic deformations of polarized varieties, Nagoya Math. J. 31 (1968), 185-245.

8. S. Mori, On a generalization of complete intersections, J. Math. Kyoto Univ. 15 (1975), 619-646.

9. A. V. Pukhlikov, Birational isomorphisms of four dimensional quintics, Invent. Math. 87 (1987), 303-329.

10. V. G. Sarkisov, Birational automorphisms of conic bundles, Math. USSR-Izv. 17 (1981), 177-202.

11. _ On the structure of conic bundles, Math. USSR-Izv. 120 (1982), 355-390.

Department of Mathematics, University of Utah, Salt lake City, Utah 84112 